\title{
$A b$ initio multi-reference perturbation theory calculations of the ground and some excited electronic states of the RbYb molecule
}

\author{
M.B. Shundalau*, A.A. Minko \\ Physics Department, Belarusian State University, 4 Nezaležnaści Ave., 220030 Minsk, Belarus
}

\section{A R T I C L E I N F O}

\section{Article history:}

Received 21 September 2016

Received in revised form 30 November 2016

Accepted 6 January 2017

Available online 9 January 2017

\section{Keywords:}

Multi-reference perturbation theory

calculations

$\mathrm{RbYb}$ molecule

Potential energy curves

Spin-orbit coupling

Vibrational states

Franck-Condon factors

\begin{abstract}
A B S T R A C T
The potential energy curves of the ground and twelve low-lying excited electronic states of the RbYb molecule have been calculated using the multi-reference perturbation theory method at the CASSCF/ XMCQDPT2 level of theory including the spin-orbit coupling. The electronic term energies, equilibrium internuclear distances, dissociation energies, transition dipole moments, the sequences of vibrational energies, the harmonic vibrational frequencies, and the Franck-Condon factors have been predicted. The potential energy curves at the asymptotic limits are in a good agreement with the experimental data for rubidium and ytterbium atoms. The obtained data would be useful for spectral experiments with RbYb molecules.
\end{abstract}

(ㄷ) 2017 Elsevier B.V. All rights reserved.

\section{Introduction}

The RbYb molecule belongs to heteronuclear diatomics, which due to the availability of an unpaired electron have both electric and magnetic permanent dipole moments. This makes possible trapping and manipulating of the weakly bound RbYb molecules in the ground $X^{2} \Sigma_{1 / 2}^{+}$state using electric as well as magnetic fields [1] enabling their usage in many applications under cold and ultracold conditions [2].

The electronic states of the RbYb molecule were studied experimentally in $[1,3]$ and theoretically in [4-8]. Some experimental difficulties preventing the formation of RbYb molecules are discussed by Tassy et al. [9] and Baumer et al. [10]. Nemitz et al. [1] obtained the energies of 15 vibrational states near the dissociation limit of the excited $1^{2} \Pi_{1 / 2}$ state of the ${ }^{87} \mathrm{Rb}^{176} \mathrm{Yb}$ and ${ }^{87} \mathrm{Rb}^{174} \mathrm{Yb}$ molecules. The energies of 7 vibrational states near the dissociation limit of the ground $X^{2} \Sigma_{1 / 2}^{+}$state of the ${ }^{87} \mathrm{Rb}^{176} \mathrm{Yb}$ molecule are determined by Münchow et al. [3].

$A b$ initio calculations of the RbYb electronic states are performed in the multi-reference CASSCF/MRCI (Complete Active Space Self-Consistent Field/Multi-Reference Configuration Interaction) $[4,6]$ and CASSCF/MRCC (Multi-Reference Coupled Cluster)

\footnotetext{
* Corresponding author.

E-mail address: shundalov@bsu.by (M.B. Shundalau).
}

$[4,5,7,8]$ approximations. It is worth mentioning that theoretical data in Refs. [4-8] for the dissociation energy $D_{e}$ of the ground $X^{2} \Sigma_{1 / 2}^{+}$state differ substantially. Sørensen et al. [4] obtained (in different basis sets and approximations) $D_{e}$ in the range $591-868 \mathrm{~cm}^{-1}$, whereas Meyer and Bohn [5] indicated only $193 \mathrm{~cm}^{-1}$. The value of the $D_{e}$ according to Tohme and Korek [6] is close to $700 \mathrm{~cm}^{-1}$. Borkowski et al. [7] in two different calculations have given 704 and $786 \mathrm{~cm}^{-1}$. Brue and Hutson [8] obtained $D_{e}=656 \mathrm{~cm}^{-1}$. The calculated equilibrium internuclear distance $R_{e}$ of the ground $X^{2} \Sigma_{1 / 2}^{+}$state is also differing: 4.89-4.67 [4], 5.41 [5], 4.99889 [6], 4.77 and 4.68 [7], $4.91 \AA ̊$ [8].

Recently [11,12] we have shown that the ab initio calculations at the CASSCF/XMCQDPT2 (Extended Multi-Configuration QuasiDegenerate 2nd Order Perturbation Theory) [13] level of theory predict the spectroscopic parameters of the ground state for the $\mathrm{KRb}$ molecule with a high accuracy. For the dissociation energy $D_{e}$ of the ground $X^{1} \Sigma^{+}$state, we have $4213.1 \mathrm{~cm}^{-1}$ that is in a better agreement with the experimental value $\left(4217.815 \mathrm{~cm}^{-1}\right.$ [14]) than those in other $a b$ initio calculations (for details see [12]). The calculated equilibrium internuclear distance of the KRb ground $X^{1} \Sigma^{+}$ state $\left(R_{e}=4.07581 \AA\right)$ also agrees considerably well with the experimental data by Pashov et al. [14] $\left(R_{e}=4.06770 \AA\right)$. We assume that the CASSCF/XMCQDPT2 level of theory is well applicable to the electronic structure calculations of heavy diatomic molecules. Especially high accuracy is expected for the ground electronic state. 
The main goals of this work are: (i) to calculate the potential energy curves (PECs) of the ground and some low-lying excited states of the $\mathrm{RbYb}$ molecule at the multi-reference perturbation level of theory including the spin-orbit coupling (SOC) and (ii) to determine other spectroscopic characteristics of the electronic states (transition dipoles, vibrational energies, Franck-Condon factors, etc.). The main attention is paid to the ground $X^{2} \Sigma_{1 / 2}^{+}$and lowlying excited $1^{2} \Pi_{1 / 2}, 1^{2} \Pi_{3 / 2}$, and $2^{2} \Sigma_{1 / 2}^{+}$states of the RbYb molecule.

\section{Computations}

The electronic configuration and the ground state term for the rubidium atom are $[\mathrm{Kr}] 5 \mathrm{~s}$ and ${ }^{2} \mathrm{~S}_{1 / 2}$, respectively. The first three excited configurations, atomic states, and their energies of the $\mathrm{Rb}$ atom are: $5 \mathrm{p} \rightarrow{ }^{2} \mathrm{P}_{1 / 2}\left(12,578.950 \mathrm{~cm}^{-1}\right),{ }^{2} \mathrm{P}_{3 / 2}\left(12,816.545 \mathrm{~cm}^{-1}\right)$; $4 \mathrm{~d} \rightarrow{ }^{2} \mathrm{D}_{5 / 2} \quad\left(19355.203 \mathrm{~cm}^{-1}\right),{ }^{2} \mathrm{D}_{3 / 2} \quad\left(19,355.649 \mathrm{~cm}^{-1}\right)$, and $6 \mathrm{~s} \rightarrow{ }^{2} \mathrm{~S}_{1 / 2}\left(20,132.510 \mathrm{~cm}^{-1}\right)$ [15]. The SOC splittings of the ${ }^{2} \mathrm{P}$ and ${ }^{2} \mathrm{D}$ terms are 237.595 and $0.446 \mathrm{~cm}^{-1}$, respectively.

The electronic configuration and the ground state term for the ytterbium atom are $[\mathrm{Xe}] 4 \mathrm{f}^{14} 6 \mathrm{~s}^{2}$ and ${ }^{1} \mathrm{~S}_{0}$, respectively. The first excited configuration, appropriate triplet and singlet terms, and energies of the states for the $\mathrm{Yb}$ atom are: $4 \mathrm{f}^{14} 6 \mathrm{~s} 6 \mathrm{p} \rightarrow{ }^{3} \mathrm{P}_{0}$ $\left(17,288.499 \mathrm{~cm}^{-1}\right),{ }^{3} \mathrm{P}_{1}\left(17,992.007 \mathrm{~cm}^{-1}\right),{ }^{3} \mathrm{P}_{2}\left(19,710.388 \mathrm{~cm}^{-1}\right)$, and ${ }^{1} \mathrm{P}\left(25068.222 \mathrm{~cm}^{-1}\right)$ [15]. The SOC splittings of the triplet ${ }^{3} \mathrm{P}$ term (703.508 and $1718.381 \mathrm{~cm}^{-1}$ ) demonstrate violation of the Landé interval rule.
The next excited term $(7 / 2,3 / 2)_{2,3,4,5}$ corresponds to the $4 f^{13} 5 d^{1} 6 s^{2}$ excited configuration with energies of the states lying within the range $23,288-28,184 \mathrm{~cm}^{-1}$ [15]. The triplet ${ }^{3} \mathrm{D}_{1,2,3}$ term originating from the $4 \mathrm{f}^{14} 5 \mathrm{~d} 6 \mathrm{~s}$ configuration with the energies ranging $24,489-25,271 \mathrm{~cm}^{-1}$ [15] and the ${ }^{1} \mathrm{P}$ term (see above) are located between the components of the $(7 / 2,3 / 2)_{2,3,4,5}$ term. Consequently, calculations of the molecular electronic states which correlate to the dissociation limits with given or higher excited configurations of the $\mathrm{Yb}$ atom require consideration of the $\mathrm{f}$ electrons in the active space. Such states are not considered here.

The PECs calculations have been performed for the doublet and quartet states corresponding to the three lowest dissociation limits: $\mathrm{Rb}(5 \mathrm{~s})+\mathrm{Yb}\left(6 \mathrm{~s}^{2}\right)$ (limit I), $\mathrm{Rb}(5 \mathrm{p})+\mathrm{Yb}\left(6 \mathrm{~s}^{2}\right)$ (limit II), and $\mathrm{Rb}$ $(5 s)+Y b\left(6 s 6 p\right.$, only triplet term) (limit III). Note that the ${ }^{2} D$ term of the $\mathrm{Rb}$ atom lies between the ${ }^{3} \mathrm{P}_{1}$ and ${ }^{3} \mathrm{P}_{2}$ components of the ${ }^{3} \mathrm{P}$ term of the $\mathrm{Yb}$ atom. Consequently, it is assumed that the molecular electronic terms corresponding to the $\mathrm{Rb}(4 \mathrm{~d})+\mathrm{Yb}\left(6 \mathrm{~s}^{2}\right)$ and $\mathrm{Rb}(6 \mathrm{~s})+\mathrm{Yb}\left(6 \mathrm{~s}^{2}\right)$ dissociation limits are strongly perturbed by the terms corresponding to the $\mathrm{Rb}(5 \mathrm{~s})+\mathrm{Yb}(6 \mathrm{~s} 6 \mathrm{p})$ dissociation limit. Since the terms corresponding to the $\mathrm{Rb}(5 \mathrm{~s})+\mathrm{Yb}$ $\left(4 \mathrm{f}^{13} 5 \mathrm{~d}^{1} 6 \mathrm{~s}^{2}\right)$ dissociation limit, which must be directly above the above-mentioned terms, are not included into the calculations, the terms corresponding to the $\mathrm{Rb}(4 \mathrm{~d})+\mathrm{Yb}\left(6 \mathrm{~s}^{2}\right)$ and $\mathrm{Rb}(6 \mathrm{~s})+\mathrm{Yb}$ $\left(6 s^{2}\right)$ dissociation limits are pushed up. Consequently, if excitations of the f-electrons of the $\mathrm{Yb}$ atom are not taken into account in the active space, PECs of these terms [6] will be of the artifact character.

Table 1

Molecular spectroscopic constants of ${ }^{85} \mathrm{Rb}^{174} \mathrm{Yb}$ molecule.

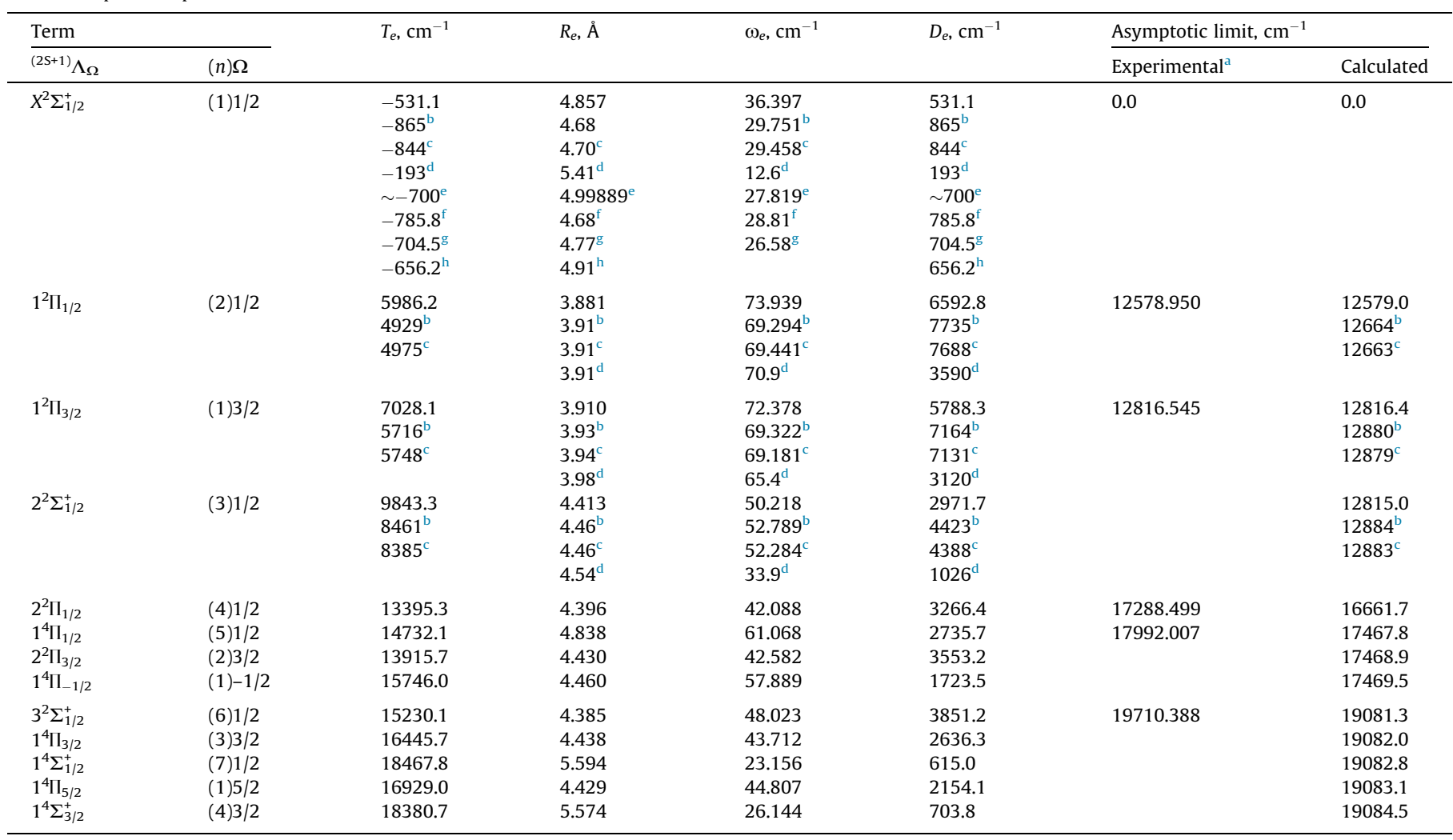

a NIST [15].

b Sørensen et al. [4] without counterpoise correction.

c Sørensen et al. [4] with counterpoise correction.

d Meyer and Bohn [5].

e Tohme and Korek [6].

f Borkowski et al. [7] with all-electron basis set.

g Borkowski et al. [7] with ECP basis set.

$\mathrm{h}$ Brue and Hutson [8]. 
The ground $X^{2} \Sigma^{+}$state corresponds to the asymptotic limit I; the doublet $2^{2} \Sigma^{+}$and $1^{2} \Pi$ states correspond to the asymptotic limit II; the doublet $3^{2} \Sigma^{+}, 2^{2} \Pi$ states, and the quartet $1^{4} \Sigma^{+}$and $1^{4} \Pi$ states correspond to the asymptotic limit III. When SOC is taken into account, the $X^{2} \Sigma^{+}, 2^{2} \Sigma^{+}$and $3^{2} \Sigma^{+}$states correspond to the $X^{2} \Sigma_{1 / 2}^{+}$, $2^{2} \Sigma_{1 / 2}^{+}$and $3^{2} \Sigma_{1 / 2}^{+}$states, respectively; the $1^{2} \Pi$ and $2^{2} \Pi$ states split into the $1^{2} \Pi_{3 / 2}, 1^{2} \Pi_{1 / 2}$ and $2^{2} \Pi_{3 / 2}, 2^{2} \Pi_{1 / 2}$ states, respectively; the $1^{4} \Sigma^{+}$state splits into the $1^{4} \Sigma_{3 / 2}^{+}$and $1^{4} \Sigma_{1 / 2}^{+}$states; the $1^{4} \Pi$ state splits into the $1^{4} \Pi_{5 / 2}, 1^{4} \Pi_{3 / 2}, 1^{4} \Pi_{1 / 2}$ and $1^{4} \Pi_{-1 / 2}$ states. Note that, since the spin-orbit coupling mixes states of different multiplicity, the ${ }^{(2 S+1)} \Lambda_{\Omega}$ designation is rather conditional in character, especially for the states belonging to the asymptotic limit III. Then $(n)$ $\Omega$ designation, where $n$ is a number of the state with the given $\Omega$, is more appropriate. The ${ }^{(2 S+1)} \Lambda_{\Omega}$ designation is used here to emphasize the relationship between the spin-mixed states and the spin-free ones, and hence such designation sometimes is useful. Both designations $\left({ }^{(2 S+1)} \Lambda_{\Omega}\right.$ and $(n) \Omega$ ) are given in Table 1 .

The small core Stuttgart RSC ECP [16] and the large core Stuttgart RLC ECP [17] for rubidium and ytterbium atoms, respectively, have been used in our calculations. The Gaussian basis sets used are: $(13 \mathrm{~s} 10 \mathrm{p} 5 \mathrm{~d} 3 \mathrm{f} 1 \mathrm{~g}) \rightarrow[8 \mathrm{~s} 7 \mathrm{p} 5 \mathrm{~d} 3 \mathrm{f} 1 \mathrm{~g}](\mathrm{ECP} 28 \mathrm{MDF})$ for rubidium atom [16] and (7s7p6d) (ECP60MDF) for ytterbium atom [17].

First, the CASSCF calculations with the state-averaged (SA) procedure were done. The active space for the CASSCF calculations was 3 electrons in 18 orbitals. The SA procedure was realized for 5 doublet and 2 quartet states. The $\operatorname{SA}-\operatorname{CASSCF}(3,18)$ calculations were performed pointwisely by steps of $0.05,0.10,0.25$, and $0.50 \AA$ for the internuclear distances ranging 3.00-6.00, 6.00$8.00,8.00-9.00$, and 9.00-20.00 Å, respectively.

Then the calculations for doublet and quartet states were performed in the XMCQDPT2 [13] approximation. All the eight lowest double occupied orbitals were included in the perturbation based calculations. The ISA shift of 0.048 [18] was used for the present approach. The dimensions of the effective Hamiltonian are: $9 \times 9$ and $5 \times 5$ for doublet and quartet states, respectively.
Finally, the spin-orbit coupling (SOC) calculations were performed with the one-electron Pauli-Breit operator. The effective nuclear charges (see, for example, [19-21]) are: 2035.0 and 8925.0 for the rubidium and ytterbium atoms, respectively. Large values of $Z_{\text {eff }}$ should compensate errors of the expectation value $<1 / r^{3}$, in the small $r$ region arising with the use of the ECP basis sets [21].

So, the following parameters of the $a b$ initio calculations at the MRPT + SOC level of theory influence the calculation accuracy: a number of the states considered in the perturbation theory method [12] (i); qualitative composition $(\Sigma, \Pi, \Delta)$ of the above-mentioned states [12] (ii); the energy denominator shift (EDS) for the intruder state avoidance (ISA) [12,18] (iii), and $Z_{\text {eff }}$ values [21] (iv). These parameters allow one to vary the energies of the low-lying atomic states, and the optimal choice of these parameters provides the required accuracy for the characteristics of the low-lying electronic molecular states.

All these calculations were performed using the Firefly quantum chemical package [22]. The electronic energy terms $T_{e}$ and the equilibrium internuclear distances $R_{e}$ are obtained using the fourth degree polynomial approximation of the $a b$ initio PECs near their minima.

The transition dipole moments (TDMs) are computed based on the CASSCF functions at the CASSCF/XMCQDPT2 + SOC level of theory. The calculations of the TDMs are performed using the GAMESS quantum chemical package [23].

The calculations of the vibrational states energies and the Franck-Condon factors are performed using the LEVEL program package [24].

\section{Results and discussion}

\subsection{Electronic states}

The calculated PECs versus internuclear distances are shown in Fig. 1 and are also given in the supplementary material. The calcu-

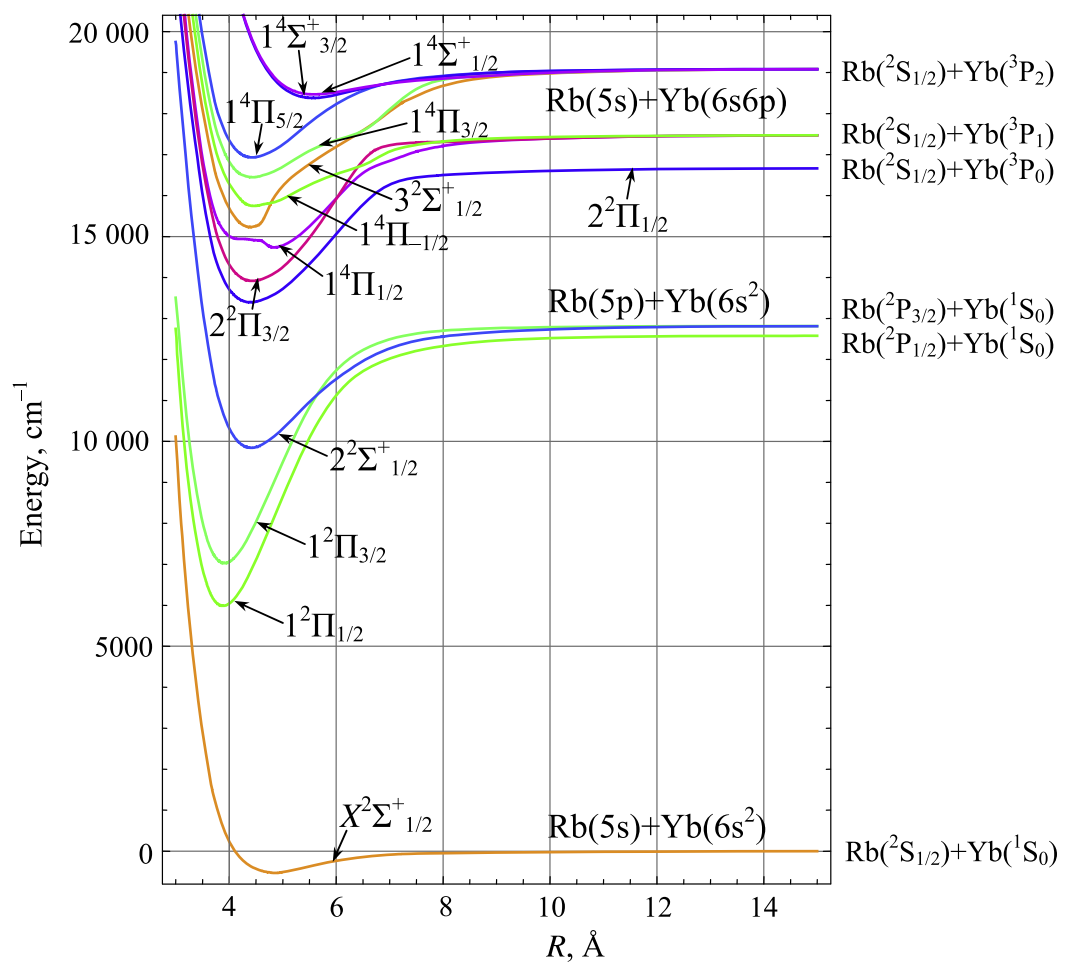

Fig. 1. The low-lying terms of the RbYb molecule calculated at the CASSCF(3,18)/XMCQDPT2 + SOC level of theory. 
lated molecular spectroscopic constants (the electronic term energy $T_{e}$, the equilibrium internuclear distance $R_{e}$, the dissociation energy $D_{e}$, and the harmonic vibrational frequency $\omega_{e}$ ) are presented and compared with the previous theoretical results in Table 1.

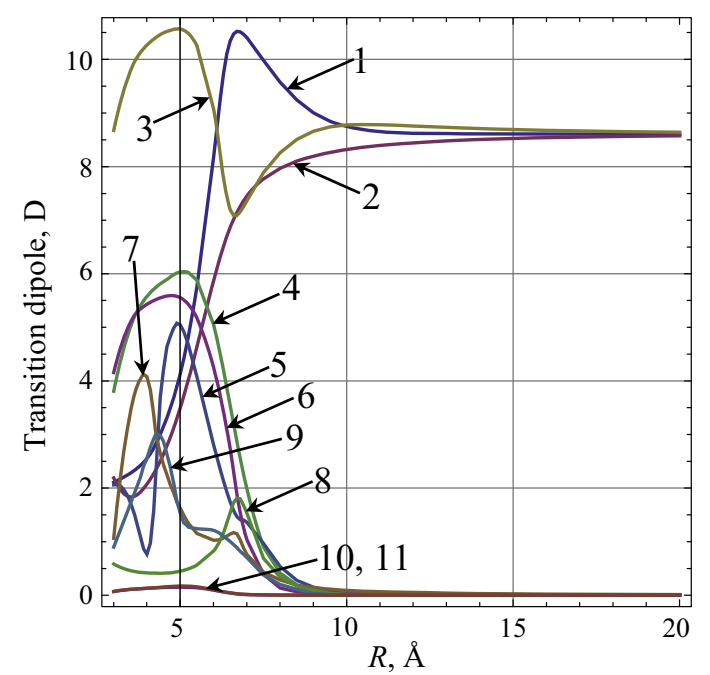

Fig. 2. Calculated transition dipole moments for the transitions from the ground (1) $1 / 2$ state to the states (2)1/2 (1), (1)3/2 (2), (3) $1 / 2$ (3), (4) $1 / 2$ (4), (5) $1 / 2$ (5), (2) $3 / 2$ (6), (1) $-1 / 2$ (7), (3)3/2 (8), (6)1/2 (9), (7) $1 / 2$ (10), and (4)3/2 (11).

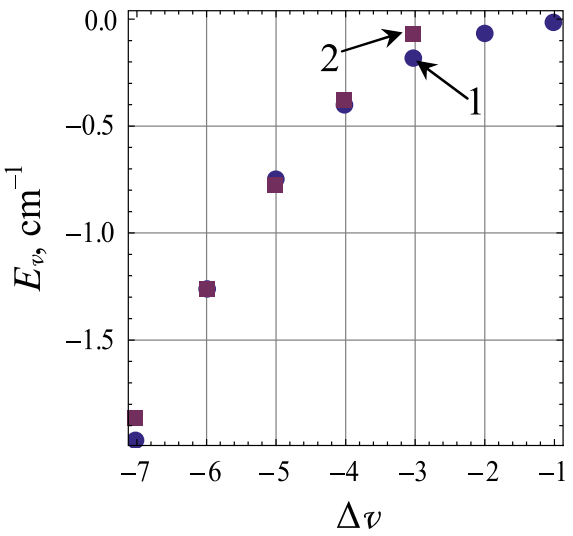

$a$

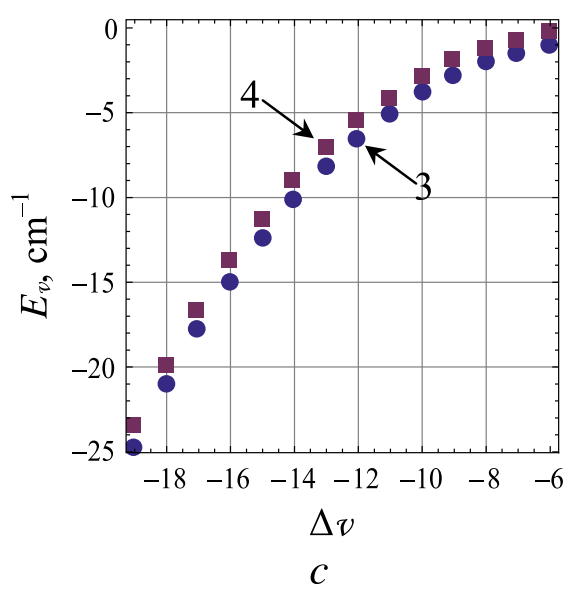

For the PECs of the asymptotic limit II at the internuclear distance of $20 \AA$, we have 12579.0, 12815.0, and $12816.4 \mathrm{~cm}^{-1}$ $\left(1^{2} \Pi_{1 / 2}, 2^{2} \Sigma_{1 / 2}^{+}\right.$, and $1^{2} \Pi_{3 / 2}$ states, respectively) with the SOC splitting that equals $237.4 \mathrm{~cm}^{-1}$. As can be seen from Table 1 , these values are in a good agreement with the NIST [15] experimental data $\left(12578.950,12816.545\right.$ and $\left.237.595 \mathrm{~cm}^{-1}\right)$. This fact can be a criterion pointing to the adequacy of the determined spectroscopic parameters for the ground $X^{2} \Sigma_{1 / 2}^{+}$and excited $1^{2} \Pi_{1 / 2}, 2^{2} \Sigma_{1 / 2}^{+}$, and $1^{2} \Pi_{3 / 2}$ states.

Our calculated molecular constants $D_{e}$ and $R_{e}$ for the ground electronic state $X^{2} \Sigma_{1 / 2}^{+}\left(D_{e}=531.1 \mathrm{~cm}^{-1}, R_{e}=4.857 \AA\right)$ are approximately intermediate between the results of Sørensen et al. [4] $\left(865 \mathrm{~cm}^{-1}, 4.68 \AA\right)$ and Meyer and Bohn [5] (193 $\left.\mathrm{cm}^{-1}, 5.41 \AA\right)$, being the closest to the data of Brue and Hutson [8] $\left(656.2 \mathrm{~cm}^{-1}, 4.91 \AA\right)$.

Our calculated $T_{e}$ values of the $1^{2} \Pi_{1 / 2}, 2^{2} \Sigma_{1 / 2}^{+}$and $1^{2} \Pi_{3 / 2}$ states are higher than those of Sørensen et al. [4]. And, since the asymptotic behavior of these PECs in our and Sørensen's [4] calculations differs slightly, our calculated $D_{e}$ values are lower than those of Sørensen et al. [4] (see Table 1). The equilibrium internuclear distances of the PECs of $1^{2} \Pi_{1 / 2}, 2^{2} \Sigma_{1 / 2}^{+}$and $1^{2} \Pi_{3 / 2}$ states obtained in our calculations are similar to the values of Sørensen et al. [4].

PECs of the asymptotic limit III are underestimated approximately by $626 \mathrm{~cm}^{-1}$ relative to the experimental energies [15] and the calculated total SOC splitting $\left(2422.8 \mathrm{~cm}^{-1}\right)$ of this limit corresponds to the experimental [15] value $\left(2421.889 \mathrm{~cm}^{-1}\right)$.

The calculated TDMs for the transitions from the ground state to the all spin-mixed excited states with the $\Delta \Omega=0, \pm 1$ selection rules as the functions of internuclear distances are shown in Fig. 2. Near the dissociation limits only TDMs in the case of the

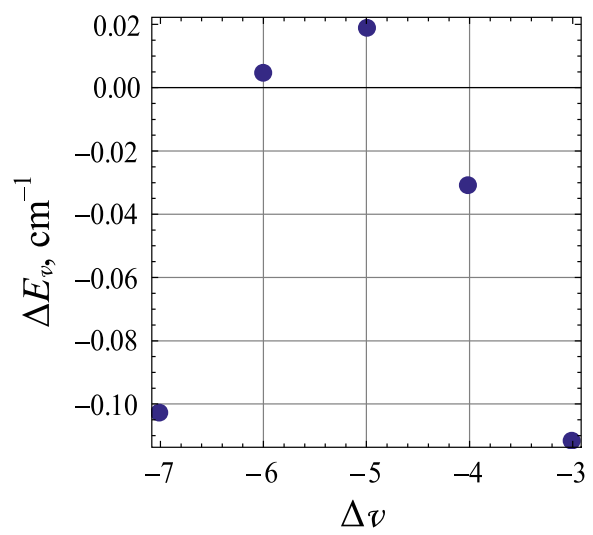

$b$

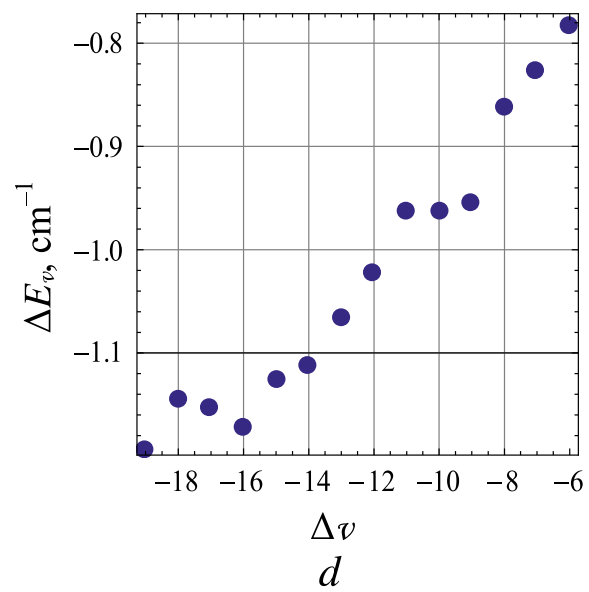

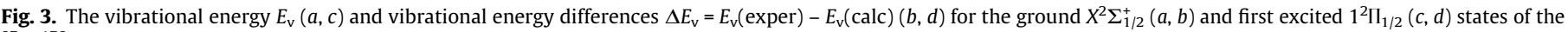
${ }^{87} \mathrm{Rb}^{176} \mathrm{Yb}$ molecule: (1) Münchow et al. [3], (3) Nemitz et al. [1], (2), and (4) the present calculations (see details in the text). 
limit II are nonzero in conformity with the selection rules for the atomic transitions.

\subsection{Vibrational states}

The ${ }^{85} \mathrm{Rb}^{174} \mathrm{Yb}$ molecule is the most abundant isopotomer (23\%) among 14 natural isotopomers of the title molecule. Thus, the vibrational energies for the ${ }^{85} \mathrm{Rb}^{174} \mathrm{Yb}$ molecule (and some other isotopomers) are calculated for all spin-mixed PECs up to the dissociation limit of the respective state, and the harmonic vibrational frequencies are also obtained (see Table 1 ). For the ground $X^{2} \Sigma_{1 / 2}^{+}$ and excited $1^{2} \Pi_{1 / 2}, 1^{2} \Pi_{3 / 2}$, and $2^{2} \Sigma_{1 / 2}^{+}$states we have $46,168,143$ and 126 vibrational levels, respectively. For all of these states, the obtained $\omega_{e}$ values are the largest among all the earlier calculated values (see Table 1).

Since the experimental data for the vibrational states of the $\mathrm{RbYb}$ molecule are few in number, we compare the calculated energies of the weakly-bound vibrational states for the ground and for the first excited electronic states near the dissociation limits with the experimental data of Münchow et al. [3] and Nemitz et al. [1] in the case of ${ }^{87} \mathrm{Rb}^{176} \mathrm{Yb}$ isotopomer. The results are presented in Fig. 3. Here $\Delta v=v-v_{\max }$ is the relative vibrational quantum number and $\mathrm{v}_{\max }$ is the vibrational quantum number of the last bound state.

The ground state PEC has a shallow well and hence PEC has a sloping behavior near the dissociation limit. Since our calculations are limited to $20 \AA$, a faster convergence of the vibrational states to the dissociation limit than that observed in the experiment is predicted [3]. So, the calculated energy of the $v_{\max }$ vibrational state is artificially overestimated relative to the lower vibrational states. In Fig. $3 \mathrm{a}$ and b we compare the calculated energies with $\Delta \mathrm{v}$ and the corresponding experimental values with $\Delta v-2$. For the first excited $1^{2} \Pi_{1 / 2}$ state, a more exact agreement between the calculated and experimental [1] vibrational energies for the same $\Delta \mathrm{v}$ values is observed (Fig. $3 \mathrm{c}$ and d).

\subsection{Franck-Condon factors}

Basing on our $a b$ initio PECs and calculated vibrational eigenfunctions, we predict the Franck-Condon factors (FCFs) between the relevant states of the ${ }^{85} \mathrm{Rb}^{174} \mathrm{Yb}$ molecule. FCFs have been calculated for the rovibronic transitions between the all spin-mixed electronic states with the $\Delta \Omega=0, \pm 1$ selection rules. These calculations are performed for the transitions between the fixed vibrational level $v^{\prime}$ of the upper electronic state and a sequence of

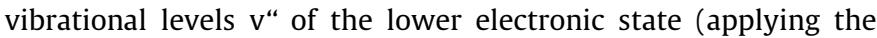
selection rules $\Delta J=0$ or $\Delta J= \pm 1$ ).

The selected FCFs between the vibrational levels of the first three exited states and the vibrational levels of the ground state with $\Delta J=0$ selection rule are presented in Fig. 4. FCFs shown here are calculated for the transitions from the lowest vibrational levels $\left(v^{\prime}=0 \ldots 4\right)$ of the excited electronic states (Fig. 4a, d, g) (1), for the
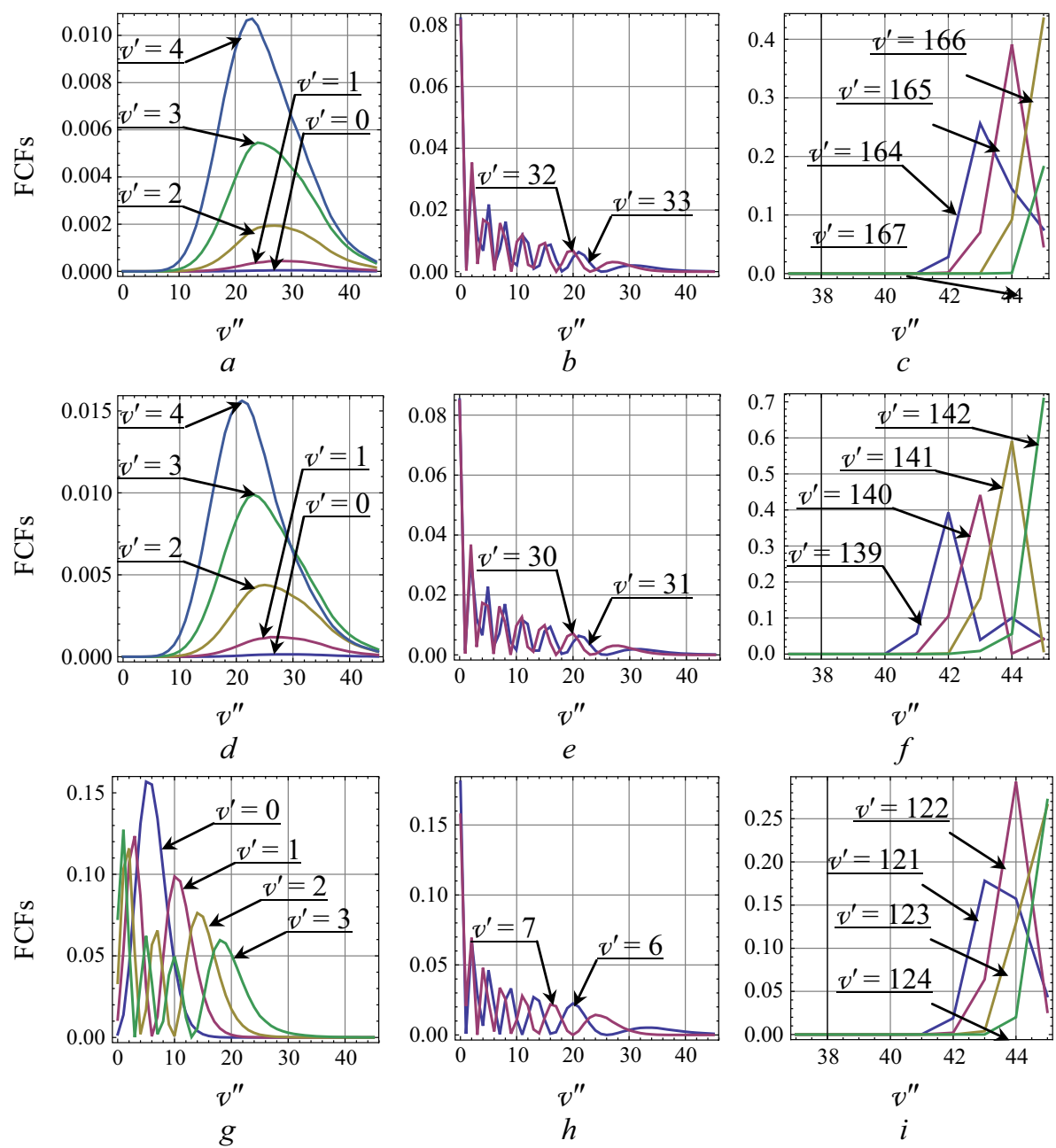

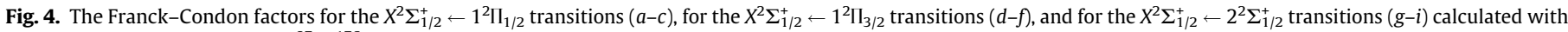
the $\Delta J=0$ selection rule for the ${ }^{87} \mathrm{Rb}^{176} \mathrm{Yb}$ molecule. 
transitions with the largest FCFs to the ground rovibronic $X^{2} \Sigma_{1 / 2}^{+}$ $\left(v^{\prime \prime}=0, J=0\right)$ state (Fig. 4 b, e, $\left.h\right)(2)$, and for the transitions with the largest values of all possible FCFs (Fig. 4c, f, $i$ ) (3).

The FCFs distributions for the $X^{2} \Sigma_{1 / 2}^{+} \leftarrow 1^{2} \Pi_{1 / 2}$ and $X^{2} \Sigma_{1 / 2}^{+} \leftarrow 1^{2} \Pi_{3 / 2}$ transitions have a similar character (see Fig. 4a-f). The largest values of FCFs for the transitions to the "absolute" ground state $X^{2} \Sigma_{1 / 2}^{+}\left(\mathrm{v}^{\prime \prime}=0, J=0\right)$ are about 0.08 with $\mathrm{v}^{\prime}=28 \ldots 34$. Note that the rovibronic transitions from the weakly-bound vibrational states near the dissociation limits of the excited electronic states $\left(v^{\prime}=160 \ldots 166\right.$ for $1^{2} \Pi_{1 / 2}$ state and $v^{\prime}=135 \ldots 142$ for $1^{2} \Pi_{3 / 2}$ state) to similar vibrational states of the ground electronic state $\left(\mathrm{v}^{\prime \prime}=40 \ldots 46\right)$ are most probable.

For the $X^{2} \Sigma_{1 / 2}^{+} \leftarrow 2^{2} \Sigma_{1 / 2}^{+}$transitions to the "absolute" ground state $X^{2} \Sigma_{1 / 2}^{+}\left(\mathrm{v}^{\prime \prime}=0, J=0\right)$, the largest FCFs correspond to the transitions from the $2^{2} \Sigma_{1 / 2}^{+}\left(v^{\prime}=4 \ldots 10\right)$ vibronic states with FCFs on the order of 0.1 . Taking into account that the predicted average value of TDM for the $2^{2} \Sigma^{+} \leftarrow X^{2} \Sigma^{+}((3) 1 / 2 \leftarrow(1) 1 / 2)$ transition is about 8.5 D over the whole $3.5-20.0 \AA$ region (see Fig. 2), the $2^{2} \Sigma_{1 / 2}^{+}((3)$ $1 / 2$ ) state is the best candidate for the optical scheme for the formation of $\mathrm{RbYb}$ molecules in the ground rovibronic state [4].

\section{Conclusions}

We have calculated the potential energy curves for the ground and twelve low-lying excited states of the RbYb molecule at the $\operatorname{CASSCF}(3,18) / \mathrm{XMCQDPT} 2+$ SOC level of theory. We have predicted the molecular spectroscopic constants and other characteristics for all of the above-mentioned states. Since at the $\operatorname{Rb}(5 \mathrm{p})+\mathrm{Yb}\left(6 \mathrm{~s}^{2}\right)$ dissociation limit our calculated PECs are in a good agreement with the experimental [15] energies and since our earlier calculations for the KRb molecule [12] in a similar approximation predict the spectroscopic parameters of the ground state of the KRb molecule with a high accuracy, we assume that the results of our calculations for the ground and low-lying excited states of the RbYb molecule are also close to the realistic values. The obtained data seem useful for spectral experiments with RbYb molecules.

\section{Acknowledgments}

This work has been supported by the State Committee on Science and Technology of the Republic of Belarus (Nr. F14LAT060). The authors are grateful to Dr. Alexandra Freidzon (Photochemistry Center of the Russian Academy of Sciences, Moscow, Russia), Dr. Alexander Granovsky (Firefly Project, Moscow, Russia), and Prof. Ruvin Ferber (Laser Centre of the University of Latvia, Riga, Latvia) for their helpful discussions.

\section{Appendix A. Supplementary material}

Supplementary data associated with this article can be found, in the online version, at http://dx.doi.org/10.1016/j.comptc.2017.01. 008.

\section{References}

[1] N. Nemitz, F. Baumer, F. Münchow, S. Tassy, A. Görlitz, Production of heteronuclear molecules in an electronically excited state by photoassociation in a mixture of ultracold Yb and Rb, Phys. Rev. A 79 (2009) 061403.

[2] S. Kotochigova, A. Petrov, M. Linnik, J. Kłos, P.S. Julienne, Ab initio properties of Li-group-II molecules for ultracold matter studies, J. Chem. Phys. 135 (2011) 164108.

[3] F. Münchow, C. Bruni, M. Madalinski, A. Görlitz, Two-photon photoassociation spectroscopy of heteronuclear YbRb, Phys. Chem. Chem. Phys. 13 (2011) $18734-18737$.

[4] L.K. Sørensen, S. Knecht, T. Fleig, C.M. Marian, Four-component relativistic coupled cluster and configuration interaction calculations on the ground and excited states of the RbYb molecule, J. Phys. Chem. A 113 (2009) 12607-12614.

[5] E.R. Meyer, J.L. Bohn, Electron electric-dipole-moment searches based on alkali-metal- or alkaline-earth-metal-bearing molecules, Phys. Rev. A 80 (2009) 042508.

[6] S.N. Tohme, M. Korek, Electronic structure and rovibrational calculation of the low-lying states of the RbYb molecule, Chem. Phys. 410 (2013) 37-44.

[7] M. Borkowski, P.S. Żuchowski, R. Ciuryło, P.S. Julienne, D. Kędziera, Ł. Mentel, P. Tecmer, F. Münchow, C. Bruni, A. Görlitz, Scattering lengths in isotopologues of the RbYb system, Phys. Rev. A 88 (2013) 052708.

[8] D.A. Brue, J.M. Hutson, Prospects of forming ultracold molecules in ${ }^{2} \Sigma$ states by magnetoassociation of alkali-metal atoms with Yb, Phys. Rev. A 87 (2013) 052709.

[9] S. Tassy, N. Nemitz, F. Baumer, C. Höhl, A. Batär, A. Görlitz, Sympathetic cooling in a mixture of diamagnetic and paramagnetic atoms, J. Phys. B: At. Mol. Opt. Phys. 43 (2010) 205309.

[10] F. Baumer, F. Münchow, A. Görlitz, S.E. Maxwell, P.S. Julienne, E. Tiesinga, Spatial separation in a thermal mixture of ultracold ${ }^{174} \mathrm{Yb}$ and ${ }^{87} \mathrm{Rb}$ atoms, Phys. Rev. A 83 (2011) 040702.

[11] M.B. Shundalau, A.A. Minko, Determination of the optimal energy denominator shift parameter of KRb electronic states in quantum chemical computations using perturbation theory, J. Appl. Spectrosc. 82 (2016) 901904.

[12] M.B. Shundalau, G.A. Pitsevich, A.E. Malevich, A.V. Hlinisty, A.A. Minko, R. Ferber, M. Tamanis, Ab initio multi-reference perturbation theory calculations of the ground and low-lying electronic states of the KRb molecule, Comp. Theor. Chem. 1089 (2016) 35-42.

[13] A.A. Granovsky, Extended multi-configuration quasi-degenerate perturbation theory: the new approach to multi-state multi-reference perturbation theory, J. Chem. Phys. 134 (2011) 214113.

[14] A. Pashov, O. Docenko, M. Tamanis, R. Ferber, H. Knöckel, E. Tiemann, Coupling of the $X^{1} \Sigma^{+}$and $a^{3} \Sigma^{+}$states of KRb, Phys. Rev. A 76 (2007) 022511.

[15] NIST Atomic Spectra Database, <http://www.nist.gov/pml/data/asd.cfm> (accessed 20.09.16).

[16] I.S. Lim, P. Schwerdtfeger, B. Metz, H. Stoll, All-electron and relativistic pseudopotential studies for the group 1 element polarizabilities from $\mathrm{K}$ to element 119, J. Chem. Phys. 122 (2005) 104103.

[17] Y. Wang, M. Dolg, Pseudopotential study of the ground and excited states of $\mathrm{Yb}_{2}$, Theor. Chem. Acc. 100 (1998) 124-133.

[18] S.-W. Chang, H.A. Witek, Choice of optimal shift parameter for the intruder state removal techniques in multireference perturbation theory, J. Chem. Theory Comp. 8 (2012) 4053-4061.

[19] W.R. Wadt, An approximate method to incorporate spin-orbit effects into calculations using effective core potentials, Chem. Phys. Lett. 89 (1982) 245248.

[20] S. Koseki, M.W. Schmidt, M.S. Gordon, MCSCF/6-31G(d, p) calculations of oneelectron spin-orbit coupling constants in diatomic molecules, J. Phys. Chem. 96 (1992) 10768-10772.

[21] S. Koseki, M.W. Schmidt, M.S. Gordon, N. Matsunaga, Main group effective nuclear charges for spin-orbit calculations, J. Phys. Chem. 99 (1995) 12764 12772.

[22] Alex A. Granovsky. Firefly version 8, <http://classic.chem.msu.su/gran/firefly/ index.html> (accessed 20.09.16).

[23] M.W. Schmidt, K.K. Baldridge, J.A. Boatz, S.T. Elbert, M.S. Gordon, J.H. Jensen, S Koseki, N. Matsunaga, K.A. Nguyen, S.J. Su, T.L. Windus, M. Dupuis, J.A. Montgomery, General atomic and molecular electronic structure system, J. Comp. Chem. 14 (1993) 1347-1363.

[24] R.J. Le Roy, Level 8.0: a computer program for solving the radial Schroedinger equation for bound and quasibound levels, University of Waterloo Chemica Physics Research Report CP-663, 2007. 